INISTIR 88-3098

\title{
MAGNETOSTATIC MEASUREMENTS FOR MINE DETECTION
}

Richard G. Geyer

National Institute of Standards and Technology

(formerly National Bureau of Standards)

U.S. Department of Commerce

Boulder, Colorado 80303-3328

October 1988

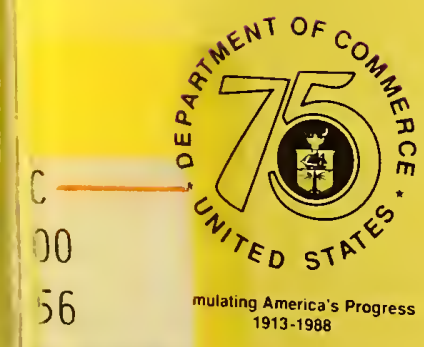

8-3098

988

$\therefore 2$ 



\title{
MAGNETOSTATIC MEASUREMENTS FOR MINE DETECTION
}

Richard G. Geyer

\author{
Electromagnetic Fields Division \\ Center for Electronics and Electrical Engineering \\ National Engineering Laboratory \\ National Institute of Standards and Technology \\ (formerly National Bureau of Standards) \\ Boulder, Colorado 80303-3328
}

October 1988

Sponsored by

U.S. Army Belvoir Research \& Development Center

Fort Belvoir, Virginia 22060-5606

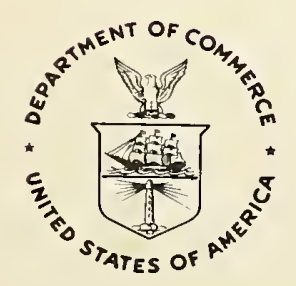

U.S. DEPARTMENT OF COMMERCE, C. William Verity, Secretary 



\section{CONTENTS}

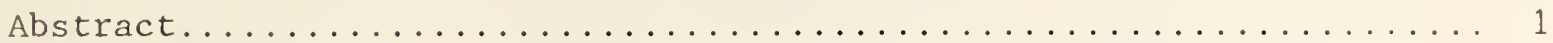

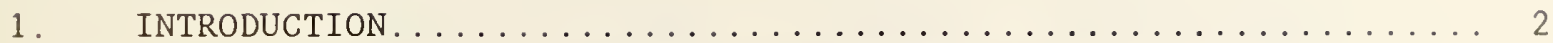

2. FACTORS AFFECTING MAGNETIC SUSCEPTIBILITY............. 2

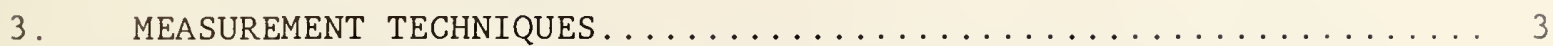

4. FREQUENCY LIMITATIONS FOR TEST MEASUREMENTS............ 8

5. SUSCEPTIBILITY MEASUREMENT OF U.S. ARMY BELVOIR MINE LANE

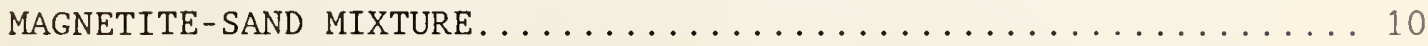

6. APPLICATION TO PASSIVE MAGNETOMETRIC DETECTION............ 13

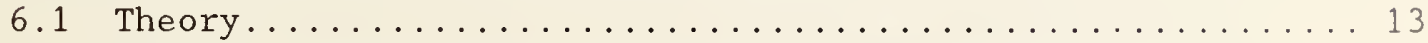

6.1.1 Magnetization Vector in Same Direction as

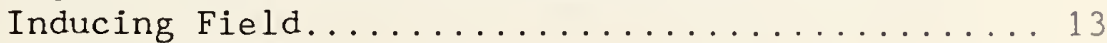

6.1.2 Magnetization Vector in a Direction Different from Inducing Field.................. 17

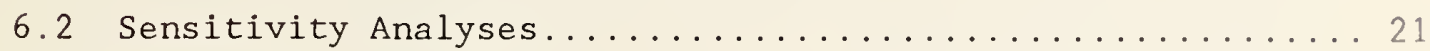

7. CONCLUSIONS ............................ 27

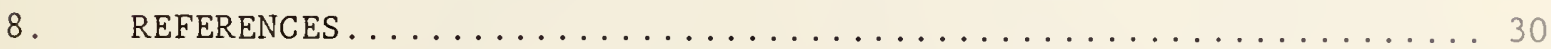





\title{
Magnetostatic Measurements for Mine Detection
}

\author{
Richard G. Geyer \\ Electromagnetic Fields Division \\ National Institute of Standards and Technology \\ Boulder, Colorado 80303
}

The use of a Maxwell inductance bridge and calibration procedure for measuring the magnetic susceptibility of magnetically linear, homogeneous, and isotropic materials are reviewed. A complication in this measurement exists since electromagnetic induction sensors respond to the product of the magnetic permeability and electrical conductivity. For this reason, frequency limitations resulting from sample size and conductivity must be considered. Such limitations can be specified by examining the in-phase and quadrature components of the induced dipole moment of a conductive, permeable sphere of diameter equivalent to that of the bridge test coil in a uniform alternating magnetic field and by choosing a maximum allowable test frequency that gives an induction number much less than 1 within the sphere.

Magnetic susceptibility measurements are applied to the passive magnetometric detection problem of an arbitrarily shaped susceptible (metallic) mine buried in a magnetically permeable earth. For analysis purposes a conservative susceptibility contrast between a typical metallic mine and host soil having the same measured magnetic characteristics as the U.S. Army Belvoir Research and Development Center (BRDC) magnetite-sand mine lane mixture was assumed. Anomalous detection limits were then calculated for various total field intensity (proton precession) sensor head heights and offset distances, given mine dimensions as small as $7.6 \mathrm{~cm}$ on a side.

Key words: inductance bridge, magnetic permeability, magnetic susceptibility, magnetometric detection, magnetostatic 
Magnetic susceptibility is a fundamental physical property of a material medium. The degree to which a body is magnetized when placed in a magnetic field is given by

$$
\overrightarrow{\mathrm{I}}=\mathrm{k} \overrightarrow{\mathrm{H}} \text {, }
$$

where $\vec{I}$ is the magnetic polarization in $A / m, \vec{H}$ is the applied magnetic field intensity in $\mathrm{A} / \mathrm{m}$ and $\mathrm{k}$ is the susceptibility (dimensionless) relating the applied field to the intensity of magnetization.

For a magnetically linear, isotropic substance, the constitutive equation relating the magnetic flux density $\vec{B}$ within a substance to the external magnetic field $\overrightarrow{\mathrm{H}}$ due to magnetization is expressed by the vector equation,

$$
\overrightarrow{\mathrm{B}}=\mu_{0}(1+\mathrm{k}) \overrightarrow{\mathrm{H}}=\mu \overrightarrow{\mathrm{H}}
$$

where $\mu_{0}$ is the permeability in vacuum $\left(4 \pi \times 10^{-7} \mathrm{H} / \mathrm{m}\right)$ and $\mathrm{k}$ is the susceptibility given in eq (1).

\section{FACTORS AFFECTING MAGNETIC SUSCEPTIBILITY}

The magnetic susceptibility of soils depends on the component magnetic minerals derived from chemical and mechanical breakdown of bedrock. Magnetic minerals of importance are few in number, and those most commonly encountered are the iron and titanium oxides which form several solid solution series in rocks (fig. 1 [1]). Depending on the fractional composition of any solid solution series, the susceptibility may vary widely (fig. 2 [2]). In addition to specific chemical composition, the susceptibility depends on grain size and the intensity of the magnetizing field. Thus, considerable variation in the susceptibility of rocks (and soils) can occur. A general description of 
typical magnetic susceptibility values for various rocks and minerals is given in Table 1 [3].

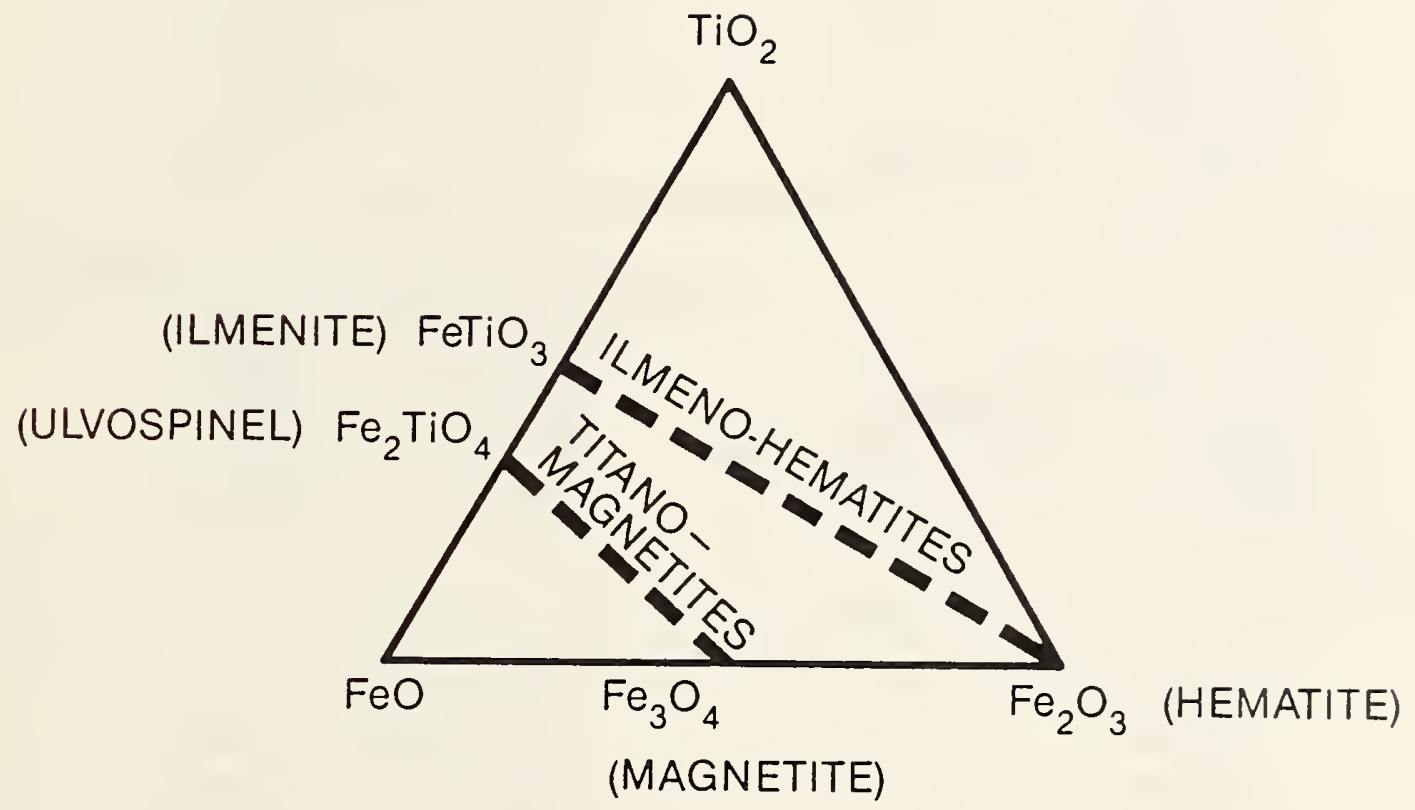

Figure 1. Composition diagram of natural magnetic minerals (after reference [1]).

\section{MEASUREMENT TECHNIQUES}

Many techniques are available for the measurement of the magnetic properties of materials. Most techniques for determining magnetic susceptibility involve placing the sample in a weak uniform and time-varying source field (less than $4 \pi \times 10^{-3} \mathrm{~A} / \mathrm{m}$ ) which does not saturate the sample so that the so-called initial susceptibility obtained is independent of the magnetizing field and hysteresis effects are avoided. As Anderson [4] notes, 
Table 1. Magnetic susceptibility of rocks and minerals (after reference [3]).

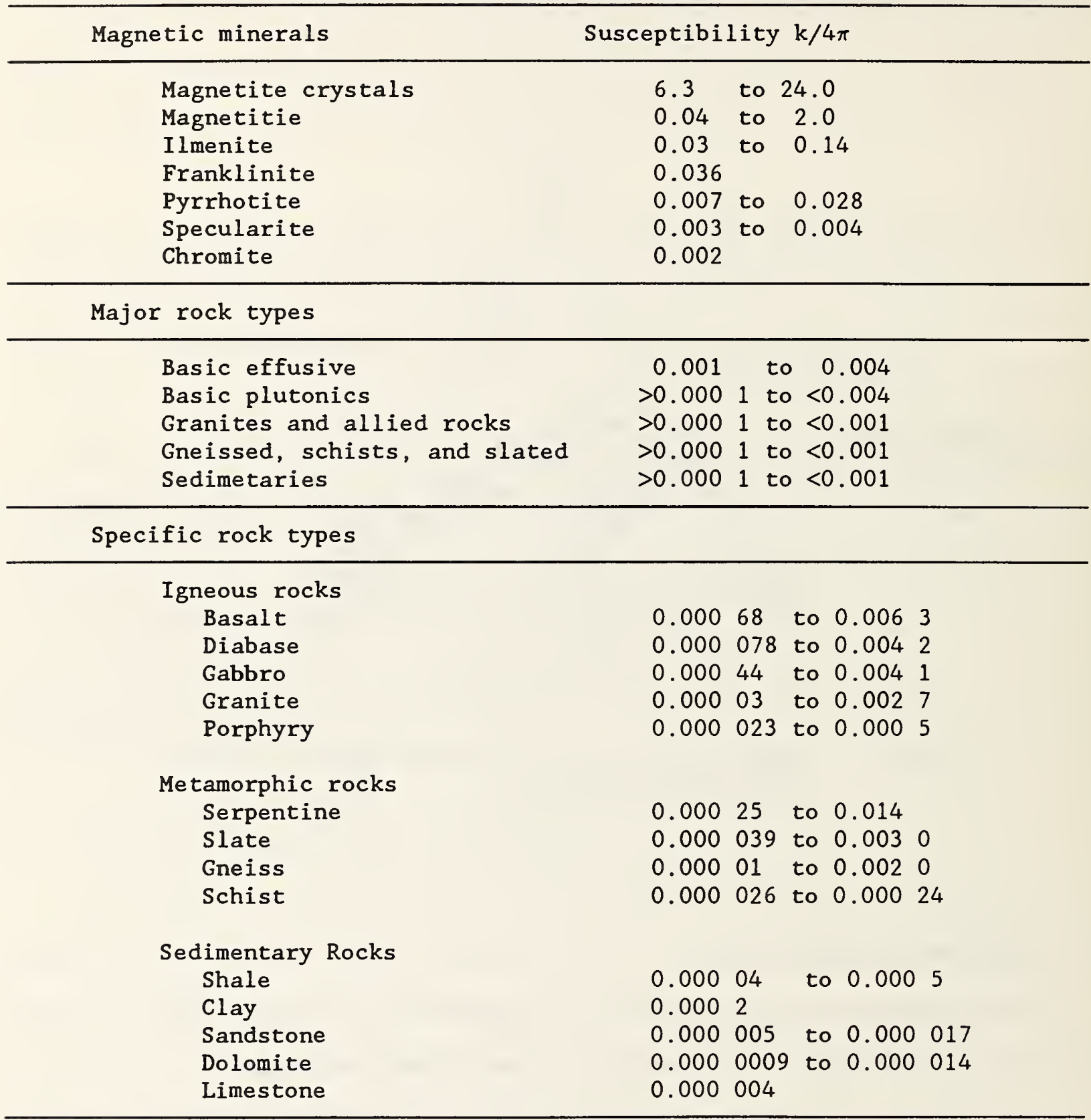

Iron ores and minerals

$\begin{array}{lll}\text { Siderite } & 0.0001 \text { to } 0.003 \\ \text { Limonite } & 0.0001 \text { to } 0.0002 \\ \text { Hematitie } & 0.00004 \text { to } 0.0001 \\ \text { Ankerite } & 0.00002 \text { to } 0.0001\end{array}$


Table 1. Magnetic susceptibility of rocks and minerals (after reference [3]) (cont.).

Typical sulfide minerals

$\begin{array}{lll}\text { Arsenopyrite } & 0.000005 \text { to } 0.0002 \\ \text { Chalcopyrite } & 0.000005 \text { to } 0.0002 \\ \text { Chromite } & 0.000005 \text { to } 0.0002 \\ \text { Markasite } & 0.000005 \text { to } 0.0002 \\ \text { Pytite } & 0.000005 \text { to } 0.0002\end{array}$

Diamagnetic minerals and rocks

Anhydrite and gypsum

Quartz

Sylvite

Calcite

Rock salt
-0.0000011 to -0.00001

-0.0000011 to -0.0000012

-0.0000009 to -0.0000011

-0.0000006 to -0.0000010

-0.0000004 to -0.0000013 


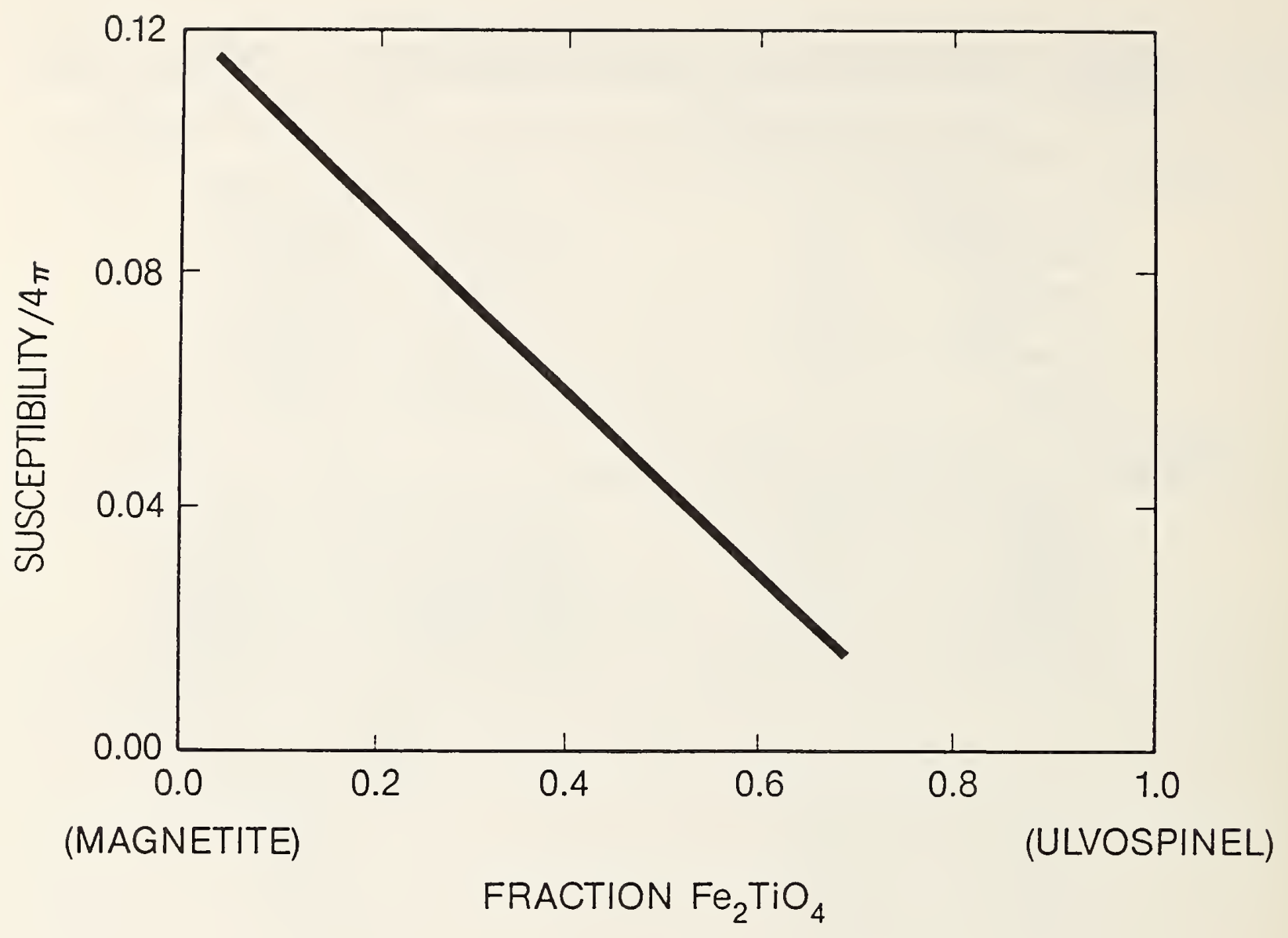

Figure 2. Susceptibility of the magnetite-ulvospinel series (after reference [2]).

measurement techniques often involve balancing a Maxwell inductance bridge with the sample inserted into one solenoidal inductance arm of the bridge (fig. 3). Another approach is to note the change in mutual reluctance between two coils set up in a coaxial or orthogonal relation to each other when the magnetic sample material is placed near the coils. The system is calibrated with a standard of known susceptibility and measurements are normalized to an equivalent half space composed of material of identical susceptibility as that of the sample. One paramagnetic standard often used for calibrating susceptibility bridge measurements is ferrous ammonium sulfate, $\mathrm{Fe}\left(\mathrm{NH}_{4}\right)_{2}$ 
$\left(\mathrm{SO}_{4}\right)_{2} \cdot 6 \mathrm{H}_{2} \mathrm{O}$, with a molecular weight of 392.15 . The susceptibility of this salt is $32.6 \times 10^{-6} \times 4 \pi$.

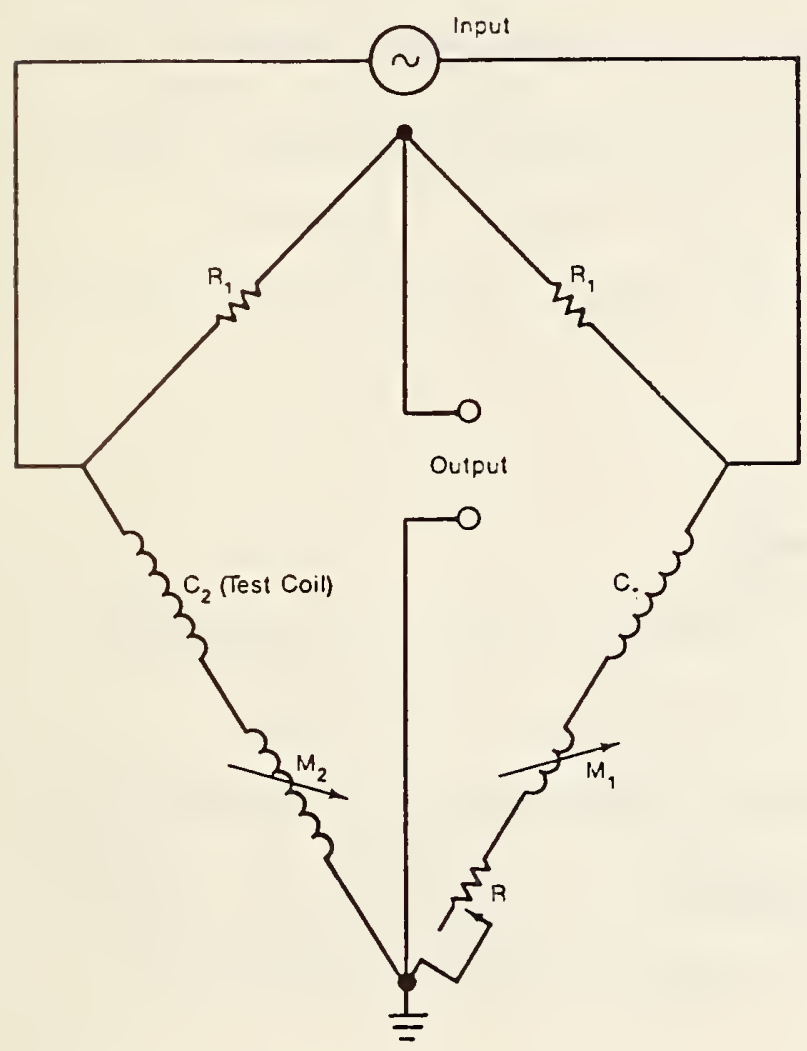

Figure 3. Typical susceptibility bridge schematic detecting inductance change in test coil $\mathrm{C}_{2}$ by action of the coil's field on a sample having unknown susceptibility.

A typical susceptibility bridge schematic is shown in figure 3 . The inductance change in test coil $\mathrm{C}_{2}$ by action of the coil's field on a sample having unknown susceptibility is measured. Coils $C_{1}$ and $C_{2}$ are solenoids carefully matched for inductance. $M_{1}$ and $M_{2}$ are identical variable inductors. In operation, the bridge would be balanced with no specimen in either coil through variable resistor $R$ and inductor $M_{1}$. The specimen is then inserted into $C_{2}$ (test coil) and the bridge balanced with inductor $M_{2}$ where the inductance adjustment is proportional to the susceptibility. Calibration of the inductance $M_{2}$ may be achieved by balancing the bridge with standard substances placed in a test tube in the coil $\mathrm{C}_{2}$ containing a known weight of a paramagnetic compound such as ferrous ammonium sulfate of known susceptibility. Calibration would be simply accomplished by balancing the bridge with inductor $M_{2}$ when the test standard is 
placed in $\mathrm{C}_{2}$. The test tube can then be removed and bridge balanced with variable inductor $M_{1}$. The same test tube with the same amount of the same standard substance is again inserted into coil $\mathrm{C}_{2}$ and the bridge balanced with $M_{2}$. This process is repeated throughout the inductance range of $C_{2}$ so as to obtain a calibration curve referenced to the known standard. If ferrous ammonium sulfate is used, it should be kept in a fresh, sealed bottle since the salt is slightly hygroscopic.

\section{FREQUENCY LIMITATIONS FOR TEST MEASUREMENTS -}

In the above techniques, the sample is energized with a low-frequency field. It is, of course, essential that this field have a frequency sufficiently low that no conductivity response of the sample will be observed. A model which provides a general rule of thumb for the highest usable frequency is that of a conducting permeable sphere in a uniform alternating magnetic field,

$$
H_{x}=H_{0} e^{-i \omega t}
$$

as shown in figure 4. Ward [5] has slightly rewritten (for $e^{-i \omega t}$ time dependence) Wait's [6] original results for the in-phase $M$ and out-of-phase $N$ components of the induced dipole moment of a sphere in a uniform alternating magnetic field when the wavelength in the external host medium is much greater than the radius of the sphere $\left(\left|\gamma_{1} a\right| \ll 1\right.$ where $\left.\gamma_{1}=\left[i \omega \mu_{1} \sigma_{1}+\omega^{2} \mu_{1} \epsilon_{1}\right]^{\frac{3}{2}}\right)$,

$$
\begin{aligned}
M-i N & =\frac{2 \mu_{2}(\tan \alpha-\alpha)-\mu_{1}\left(\alpha-\tan \alpha+\alpha^{2} \tan \alpha\right)}{2 \mu_{2}(\tan \alpha-\alpha)+2 \mu_{1}\left(\alpha-\tan \alpha+\alpha^{2} \tan \alpha\right)}, \\
\alpha & =\left(i \omega \mu_{2} \sigma_{2}\right)^{\frac{3}{2}} \mathrm{a},
\end{aligned}
$$

and $\mathrm{a}$ is the radius of the sphere in meters. 
The in-phase and quadrature components of the induced dipole moment of a sphere are shown plotted as a function of the response parameter of a sphere, $\theta=\left(\omega \sigma_{2} \mu_{2}\right)^{\frac{3}{2}} a$, in figure 5. These components are shown in parametric fashion for $\mu_{2} / \mu_{1}$ equal to 1 (free space) to $\mu_{2} / \mu_{1}$ equal to 1000 (steel sphere).

$H=H_{0} e^{-i \omega t}$
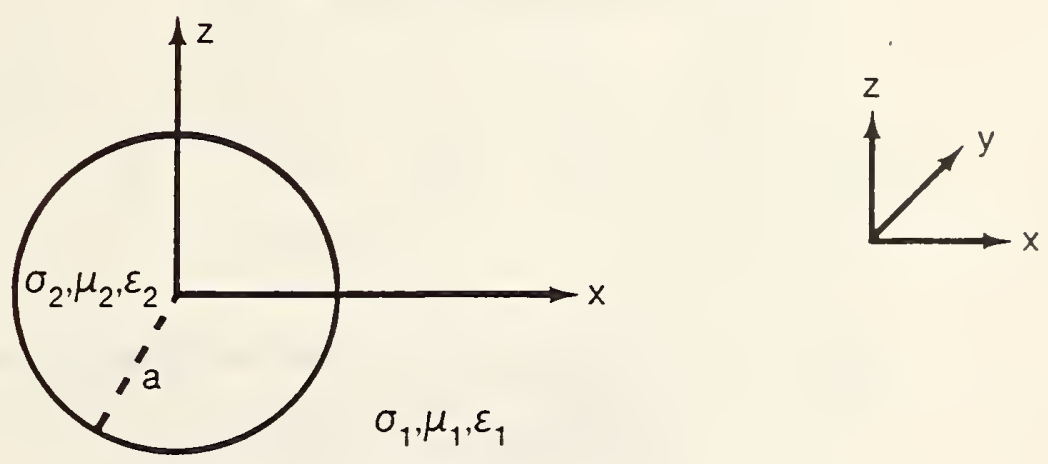

Figure 4. Conducting permeable sphere of radius a in uniform alternating magnetic field $\mathrm{H}_{\mathrm{o}} \mathrm{e}^{-1 \omega t}$. Sphere has conductivity, permeability, and permittivity of $\sigma_{2}, \mu_{2}, \epsilon_{2}$, respectively, while host medium has conductivity, permeability, and permittivity of $\sigma_{1}, \mu_{1}, \epsilon_{1}$.

In order that the conductivity response of the sample be small, we see from figure 5 that $\theta^{2}=\left(\omega \mu_{2} \sigma_{2}\right) a^{2}$ should be $\ll 1$. In the case of pure magnetite, the permeability $\mu_{2}$ is approximately $1.5 \times 4 \pi \times 10^{-7} \mathrm{H} / \mathrm{m}$ and the conductivity $\sigma_{2}$ is about $1.5 \times 10^{4} \mathrm{~s} / \mathrm{m}$. Thus, for a $2.5 \mathrm{~cm}$ diameter sphere and the condition that $\theta^{2}=0.1$, we find that the maximum allowable frequency is approximately $400 \mathrm{~Hz}$. Of course, for samples that are considerably less conductive than pure magnetite, the maximum allowable frequency may be higher while still avoiding sample conductivity response. If we ignore the effects of 


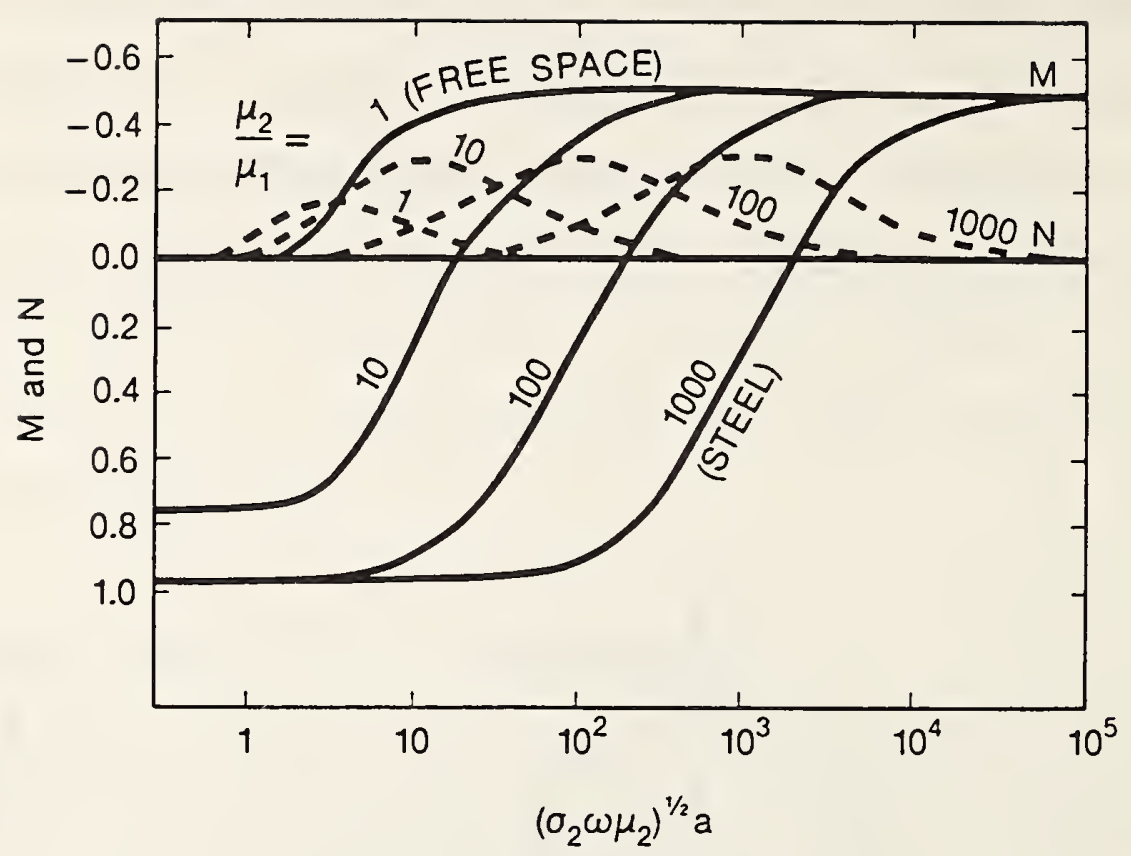

Figure 5. In-phase $M$ and out-of-phase $\mathbf{N}$ components of induced dipole moment of sphere in uniform alternating field for $\left|\gamma_{1} \alpha\right| \ll 1$.

(After reference [5]).

the air-earth interface, Wait's work [6] can also be used to quantitatively assess detection limits for both conductivity and permeability contrasts.

\section{SUSCEPTIBILITY MEASUREMENT OF U.S. ARMY BELVOIR MINE LANE MAGNETITE-SAND MIXTURE}

A susceptibility meter [7], was used in measuring the susceptibility of a sand and magnetite mix provided to NBS by Dr. Lee Anderson of BRDC. The sample consists of silica sand and magnetite of about 30 mesh size. This susceptibility meter is quite portable $(0.5 \mathrm{~kg}$ with dimensions of $190 \times 80 \times 30 \mathrm{~mm}$, operating on one disposable $9 \mathrm{~V}$ battery). 
In this meter there are two coils placed orthogonally to each other in the detector head, which is mounted in the bottom of the instrument case (circuit diagram shown in fig. 6). In a nonmagnetic environment the voltage induced in the receiver coil by the transmitter coil is zero. When a sample

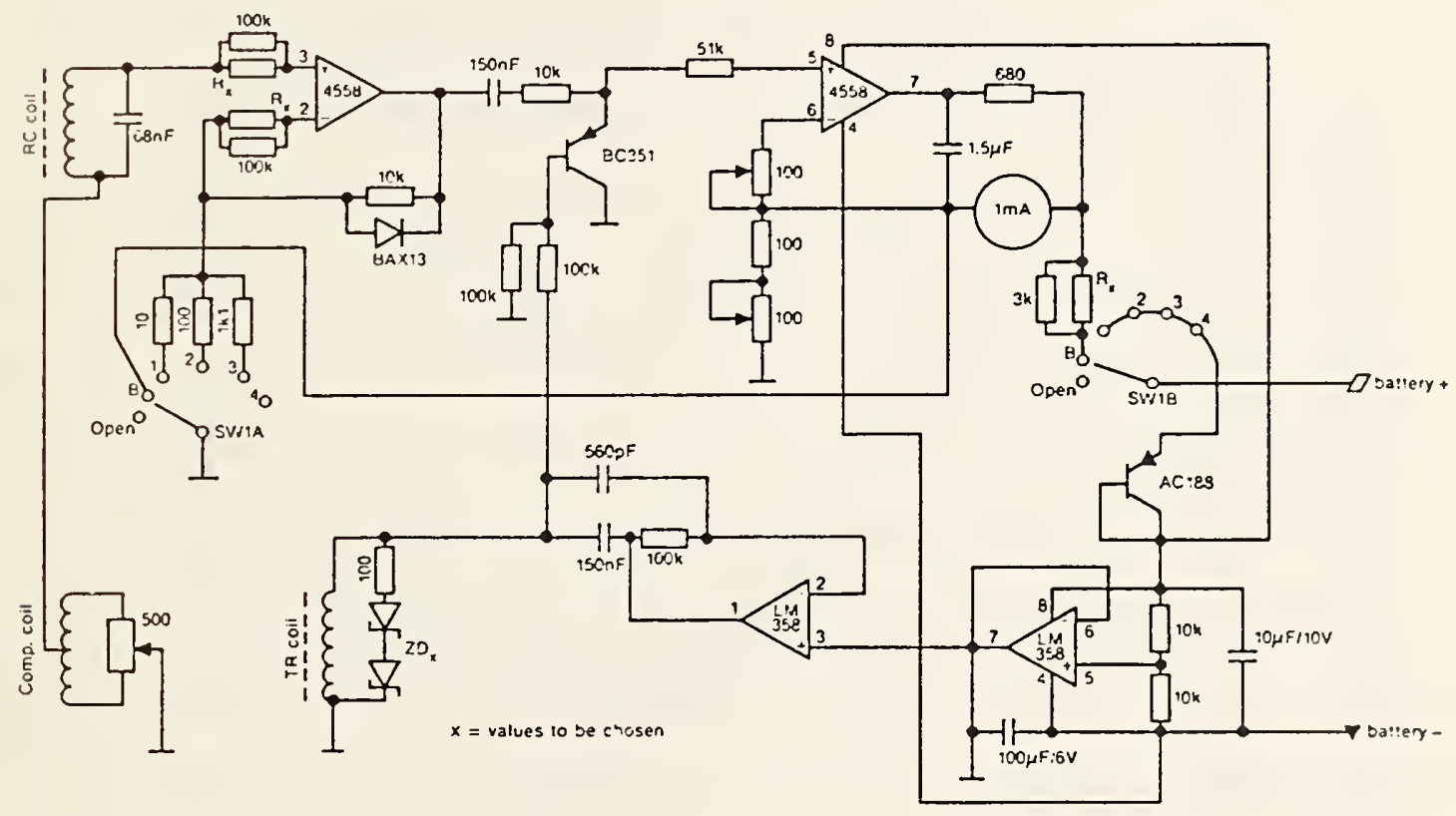

Figure 6. Circuit diagram for susceptibility meter [7].

is brought near the coils, a voltage proportional to the magnetic susceptibility of the sample is induced in the receiver coil. The received signal is detected by a phase-locked amplifier and after rectification is used to drive an analog panel meter, which is thermally compensated and directly calibrated for susceptibility. Field strengths are less than $4 \pi \times 10^{-3} \mathrm{~A} / \mathrm{m}$ at $1000 \mathrm{~Hz}$ so that with the phase sensitive receiver circuit the influence of electric conductivity in most samples is usually eliminated. As stated by Anderson [4], calibration is usually done for a half-space geometry. which is convenient when measurements are performed in the field. When 
laboratory samples are measured, a multiplicative correction factor should be applied according to the soil sample size. A chart indicating this halfspace correction factor is given in figure 7 from information provided by the manufacturer.

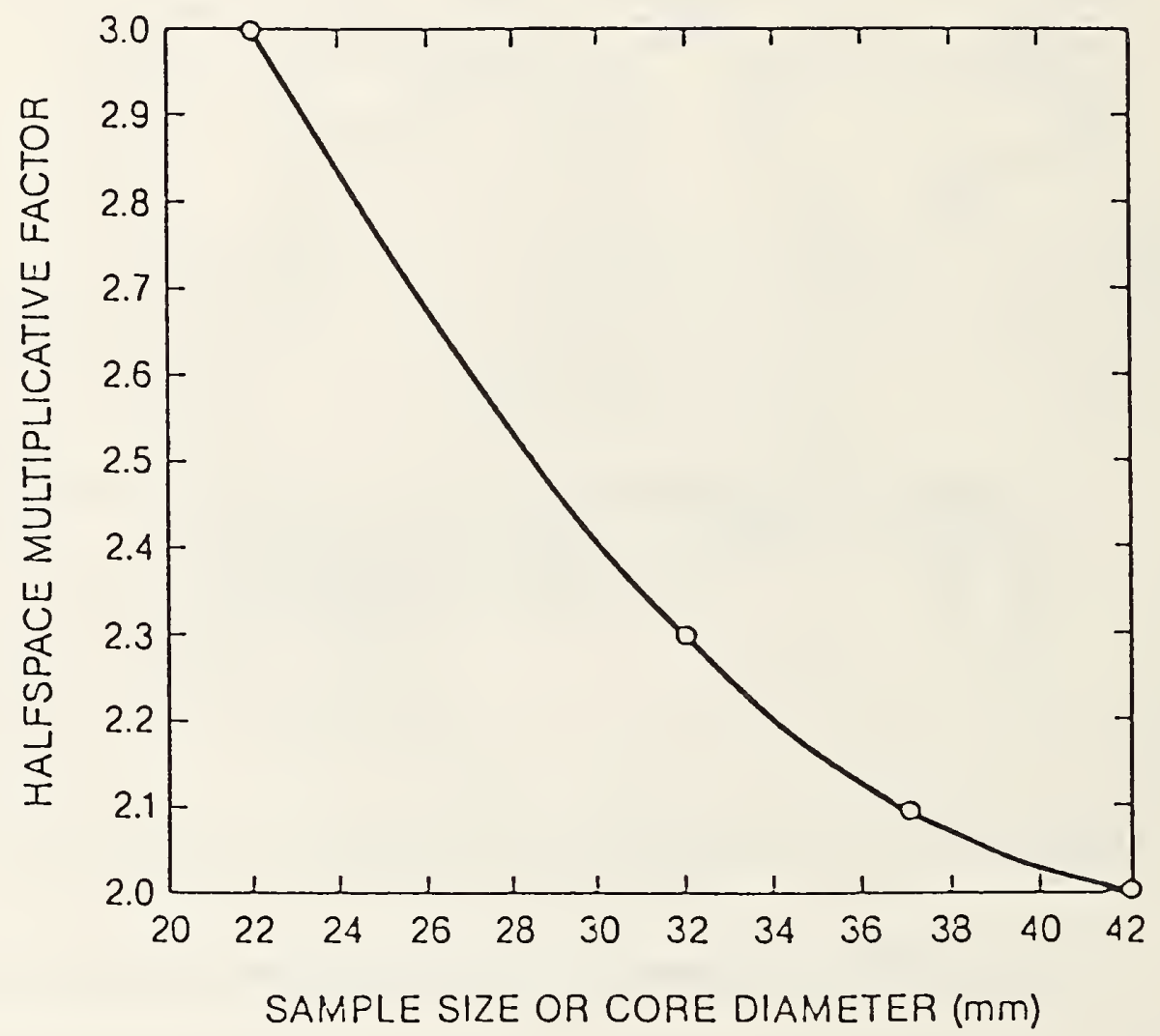

Figure 7. Half-space multiplicative correction factor for susceptibility measurements.

The silica sand-magnetite mixture's susceptibility $(\simeq 330 \mathrm{~g}$ sample, $\simeq 50 \mathrm{~mm}$ diameter) was measured and found to be approximately $175000 \times 10^{-6}$ uncorrected for sample size. After a sample size correction factor of 2.0 from figure 7 is used, the susceptibility of the $330 \mathrm{~g}$ sample is determined to be $350000 \mathrm{x}$ $10^{-6}$. For mine detection standards purposes, such measurements can provide the 
practical property range limits for which detection feasibility of various mine detection systems can be judged. Similar comments can be made about other physical property contrast limits, i.e., complex permittivity (as a function of frequency), density, and acoustic velocity.

\section{APPLICATION TO PASSIVE MAGNETOMETRIC DETECTION}

Magnetic anomaly signatures from metallic mines that can be detected passively result from magnetization of the metallic enclosure within an applied inducing (static) field. The character of the magnetic signature depends on the geometric disposition of the mine, its equivalent volume-percent magnetite content (that is, the susceptibility of the mine to magnetization) and the direction and magnitude of the inducing field. In addition, for manmade iron and steel (not stainless) objects the magnetization can also have a permanent component which is independent of the induced component so that the total magnetization is the vector sum of the two. For metallic mine detection it would be useful to examine the sensitivity of passive magnetometry to various metallic, permeable mine standards enclosed in various soil types for varying heights and offsets of the sensor head to the buried mine.

\subsection{Theory}

\subsubsection{Magnetization Vector in Same Direction as Inducing Field}

Almost any model source geometry of a metallic mine (fig. 8) may be approximated by a suitable arrangement and number of prismatic elements. Therefore a prismatic element is considered as a basic building block for passive magnetometric detection of buried metallic mines (fig. 9). 


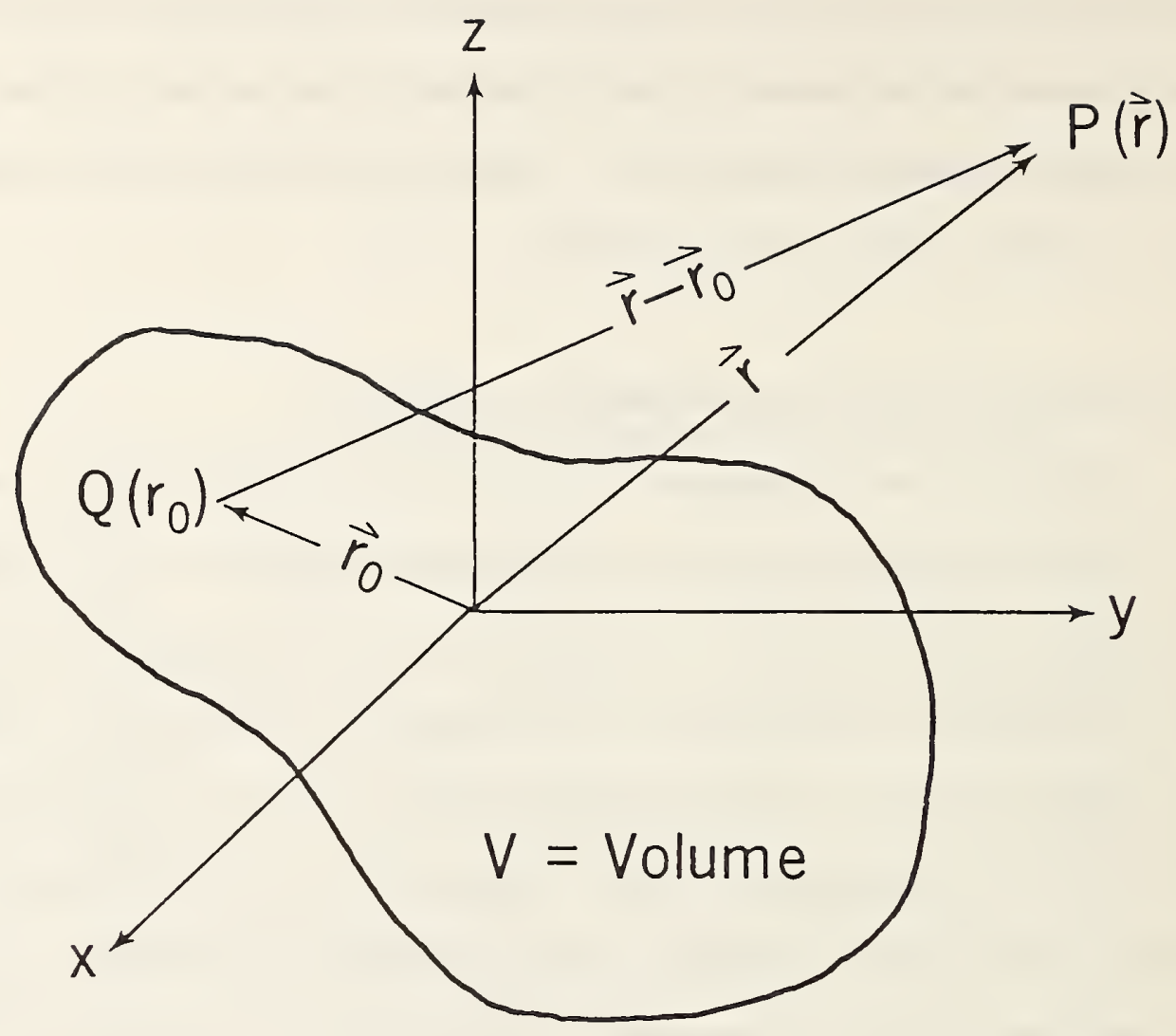

Figure 8. Arbitrary geometry of a magnetically permeable mine or firing pin as observed at a field point $P(\bar{r})$.

The expression for the total anomalous magnetic field intensity in the direction of the applied vector field, $H_{t}$, due to a prismatic model of a single metallic mine at an arbitrary field observation point $P(x, y, z)$ (that would be sensed by a typical proton precession magnetometer) is somewhat tedious to derive. Essentially,

$$
H_{t}(x, y, z)=-\frac{\partial}{\partial x} V(x, y, z) \cos I+\frac{\partial}{\partial z} V(x, y, z) \sin I
$$




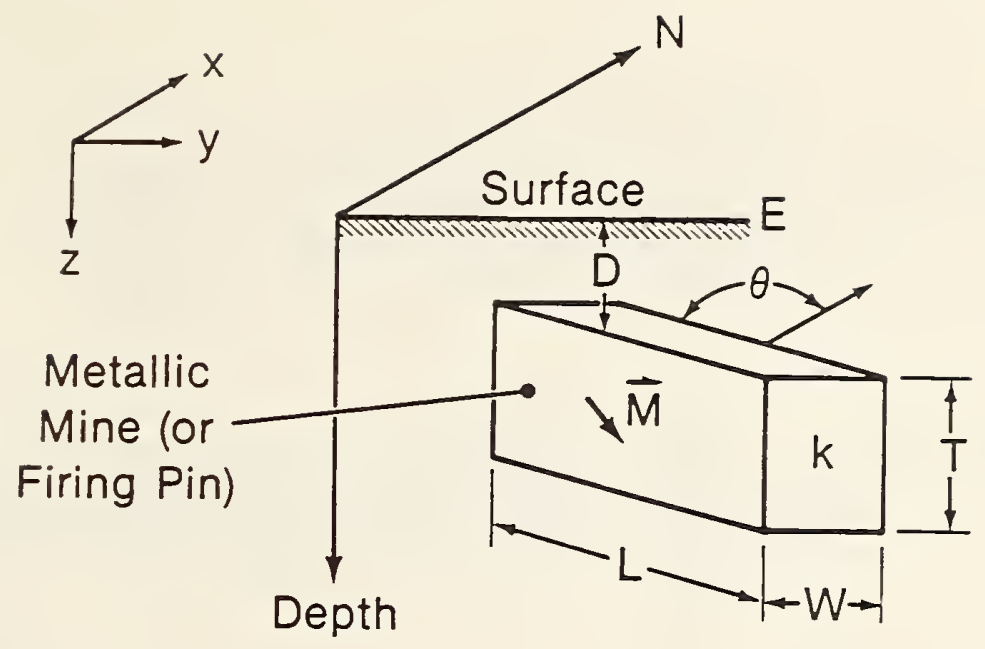

Figure 9. Geometry of prism describing metallic mine (or firing pin) relative to magnetic north at depth $D$ and having cross-sectional dimensions $\mathrm{L}$ and $\mathrm{W}$, thickness $\mathrm{T}$, and magnetic susceptibility $\mathbf{k}$.

where I is the inclination of the (earth's) applied uniform (inducing) field and where the coordinate system is configured such that $\mathrm{x}$ is directed north, $z$ is positive downward, and $V$ is the magnetic potential defined by the volume integral

$$
\begin{aligned}
& V(x, y, z)=\iiint \quad \frac{\bar{M} \cdot \bar{r}^{\prime}}{r^{\prime^{3}}} d x^{\prime} d y^{\prime} d z^{\prime} \\
& \text { volume of }
\end{aligned}
$$

where $\bar{M}$ denotes the direction and magnitude of magnetization of the prism block and $\bar{r} \bar{r}^{\prime}$ is the position vector from an element of integration at ( $x^{\prime}, y^{\prime}, z^{\prime}$ ) to an arbitrary field point $P(x, y, z)$. After much algebraic manipulation, the anomalous total magnetic field due to a single rectangular prism at any point in the $x-y$ plane, after rotation of coordinates such that coordinate surfaces coincide with the surfaces of the prism (fig. 10), may be shown to be

$H_{t}(x, y, 0)=|\bar{M}|\left\{\sin \delta \cos \delta\right.$ ln $\left[\frac{\sqrt{\left(x_{U}-x\right)^{2}+\left(y_{U}-y\right)^{2}+D_{1}^{2}}-\left(y_{U}-y\right)}{\sqrt{\left(x_{U}-x\right)^{2}+\left(y_{U}-y\right)^{2}+D_{1}^{2}}+\left(y_{U}-y\right)}\right]$ 
$-\sin \delta \cos \delta \ln \left[\frac{\sqrt{\left(\mathrm{x}_{\mathrm{U}}-\mathrm{x}\right)^{2}+\left(\mathrm{y}_{\mathrm{L}}-\mathrm{y}\right)^{2}+D_{1}^{2}}-\left(\mathrm{y}_{\mathrm{L}}-\mathrm{y}\right)}{\sqrt{\left(\mathrm{x}_{\mathrm{U}}-\mathrm{x}\right)^{2}+\left(\mathrm{y}_{\mathrm{L}}-\mathrm{y}\right)^{2}+D_{1}^{2}}+\left(\mathrm{y}_{\mathrm{L}}-\mathrm{y}\right)}\right]$

$-\sin ^{2} \delta \tan ^{-1}\left[\frac{\left(x_{U}-x\right)\left(y_{U}-y\right)}{\left(x_{U}-x\right)^{2}+D_{1} \sqrt{\left(x_{U}-x^{2}\right)+\left(y_{U}-y\right)^{2}+D_{1}^{2}}+D_{1}^{2}}\right]$

$+\sin ^{2} \delta \tan ^{-1}\left[\frac{\left(x_{U}-x\right)\left(y_{L}-y\right)}{\left(x_{U}-x\right)^{2}+D_{1} \sqrt{\left(x_{U}-x\right)^{2}+\left(y_{L}-y\right)^{2}+D_{1}^{2}}+D_{1}^{2}}\right]$

$+\cos ^{2} \delta \tan ^{-1}\left[\frac{\left(x_{U}-x\right)\left(y_{U}-y\right)}{D_{1} \sqrt{\left(x_{U}-x\right)^{2}+\left(y_{U}-y\right)^{2}+D_{1}^{2}}}\right]$

$-\cos ^{2} \delta \tan ^{-1}\left[\frac{\left(x_{U}-x\right)\left(y_{L}-y\right)}{D_{1} \sqrt{\left(x_{U}-x\right)^{2}+\left(y_{L}-y\right)^{2}+D_{1}^{2}}}\right]$

$-\sin \delta \cos \delta \ln \left[\frac{\sqrt{\left(x_{L}-x\right)^{2}+\left(y_{U}-y\right)^{2}+D_{1}^{2}}-\left(y_{U}-y\right)}{\sqrt{\left(x_{L}-x\right)^{2}+\left(y_{U}-y\right)^{2}+D_{1}^{2}}+\left(y_{U}-y\right)}\right]$

$+\sin \delta \cos \delta \ln \left[\frac{\sqrt{\left(x_{L}-x\right)^{2}+\left(y_{L}-y\right)^{2}+D_{1}^{2}}-\left(y_{L}-y\right)}{\sqrt{\left(x_{L}-x\right)^{2}+\left(y_{L}-y\right)^{2}+D_{1}^{2}}+\left(y_{L}-y\right)}\right]$

$+\sin ^{2} \delta \tan ^{-1}\left[\frac{\left(x_{L}-x\right)\left(y_{U}-y\right)}{\left(x_{L}-x\right)^{2}+D_{1} \sqrt{\left(x_{L}-x\right)^{2}+\left(y_{U}-y\right)^{2}+D_{1}^{2}}+D_{1}^{2}}\right]$ 


$$
\begin{aligned}
& -\sin ^{2} \delta \tan ^{-1}\left[\frac{\left(x_{L}-x\right)\left(y_{L}-y\right)}{\left(x_{L}-x\right)^{2}+D_{1} \sqrt{\left(x_{L}-x\right)^{2}+\left(y_{L}-y\right)^{2}+D_{1}^{2}}+D_{1}^{2}}\right] \\
& -\cos ^{2} \delta \tan ^{-1}\left[\frac{\left(x_{L}-x\right)\left(y_{U}-y\right)}{D_{1} \sqrt{\left(x_{L}-x\right)^{2}+\left(y_{U}-y\right)^{2}+D_{1}^{2}}}\right] \\
& +\cos ^{2} \delta \tan ^{-1}\left[\begin{array}{l}
\left(x_{L}-x\right)\left(y_{L}-y\right) \\
D_{1} \sqrt{\left(x_{L}-x\right)^{2}+\left(y_{L}-y\right)^{2}+D_{1}^{2}}
\end{array}\right]
\end{aligned}
$$

where $\delta=90^{\circ}$ - I. Equation (7) is an exact expression for the (static) total magnetic field at any point in the $x-y$ plane for a prism with large depth extent whose magnetization vector is in the same direction as that of inducing field and whose magnetization is uniform throughout the prism. Effects of shape demagnetization are incorporated into the value assigned to $|\bar{M}|$. The values of $x_{U}, x_{L}, y_{U}, y_{L}$ are referenced in figure 10 . Equation (7) is readily used to obtain the total field intensity for magnetized bodies of finite vertical extent (such as the magnetized mine or firing pin of interest here) by considering a prismatic body having its top at depth $D_{1}$ and its bottom at a depth $D_{2}$. The fields due to two bodies of infinite depth extent are first determined, one at depth $D_{1}$ and the other at a depth $D_{2}$. If we subtract the latter from the former, the anomalous field signature for the mine body of finite depth extent is obtained.

\subsubsection{Magnetization Vector in a Direction Different from Inducing Field}

When the magnetization vector of the subsurface metallic mine is in a direction different from that of the static inducing field (that is, when permanent magnetization is present), the following expression for the total magnetic field signature at an arbitrary point of observation $P(x, y, 0)$ in the 
$x-y$ plane due to a single prismatic element (see fig. 9) of large depth extent T results from eq (6),

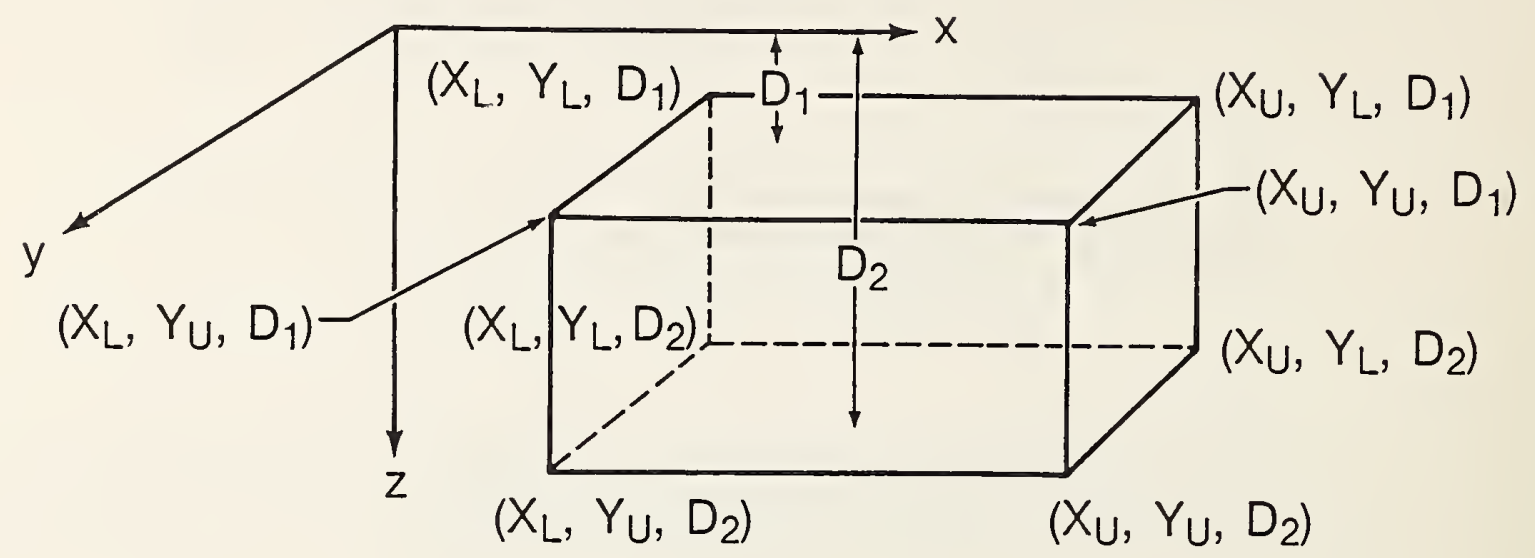

Figure 10. Rotated prismatic element whose geometry is specified by eight ordered coordinate triplets.

$H_{t}(x, y, 0)=|\bar{M}|\left\{\frac{\cos \operatorname{sinI}_{0}}{2} \sin \theta \ln \left[\frac{\sqrt{\left(x_{U}-x\right)^{2}+\left(y_{U}-y\right)^{2}+D_{1}^{2}}-\left(x_{U}-x\right)}{\sqrt{\left(x_{U}-x\right)^{2}+\left(y_{U}-y\right)^{2}+D_{1}^{2}}+\left(x_{U}-x\right)}\right]\right.$

$-\frac{\cos I \sin I_{0}}{2} \sin \theta \ln \left[\frac{\sqrt{\left(x_{L}-x\right)^{2}+\left(y_{U}-y\right)^{2}+D_{1}^{2}}-\left(x_{L}-x\right)}{\sqrt{\left(x_{L}-x\right)^{2}+\left(y_{U}-y\right)^{2}+D_{1}^{2}}+\left(x_{L}-x\right)}\right]$

$-\frac{\cos I \sin I_{0}}{2} \sin \theta \ln \left[\frac{\sqrt{\left(x_{U}-x\right)^{2}+\left(y_{L}-y\right)^{2}+D_{1}^{2}}-\left(x_{U}-x\right)}{\sqrt{\left(x_{U}-x\right)^{2}+\left(y_{L}-y\right)^{2}+D_{1}^{2}}+\left(x_{U}-x\right)}\right]$ 


$$
+\frac{\cos I \sin I_{0}}{2} \sin \theta \ln \left[\frac{\sqrt{\left(x_{L}-x\right)^{2}+\left(y_{L}-y\right)^{2}+D_{1}^{2}}-\left(x_{L}-x\right)}{\sqrt{\left(x_{L}-x\right)^{2}+\left(y_{L}-y\right)^{2}+D_{1}^{2}}+\left(x_{L}-x\right)}\right]
$$

$+\left(\cos I_{0} \sin I+\cos I \sin I_{0} \cos \theta\right) \ln \left[\frac{\sqrt{\left(x_{U}-x\right)^{2}+\left(y_{U}-y\right)^{2}+D_{1}^{2}}-\left(y_{U}-y\right)}{\sqrt{\left(x_{U}-x\right)^{2}+\left(y_{U^{-y}}\right)^{2}+D_{1}^{2}}+\left(y_{U}-y\right)}\right]$

$-\left(\cos I_{0} \sin I+\cos I \sin I_{0} \cos \theta\right) \ln \left[\frac{\sqrt{\left(x_{L}-x\right)^{2}+\left(y_{U}-y\right)^{2}+D_{1}^{2}}-\left(y_{U}-y\right)}{\sqrt{\left(x_{L}-x\right)^{2}+\left(y_{U}-y\right)^{2}+D_{1}^{2}}+\left(y_{U}-y\right)}\right]$

$-\left(\cos I_{0} \sin I+\cos I \sin I_{0} \cos \theta\right) \ln \left[\frac{\sqrt{\left(x_{U}-x\right)^{2}+\left(y_{L}-y\right)^{2}+D_{1}^{2}}-\left(y_{L}-y\right)}{\sqrt{\left(x_{U}-x\right)^{2}+\left(y_{L}-y\right)^{2}+D_{1}^{2}}+\left(y_{L}-y\right)}\right]$

$+\left(\cos I_{0} \sin I+\cos I \sin I_{0} \cos \theta\right) \ln \left[\frac{\sqrt{\left(x_{L}-x\right)^{2}+\left(y_{L}-y\right)^{2}+D_{1}^{2}}-\left(y_{L}-y\right)}{\sqrt{\left(x_{L}-x\right)^{2}+\left(y_{L}-y\right)^{2}+D_{1}^{2}}+\left(y_{L}-y\right)}\right]$

$-\cos I \cos I_{0} \sin \theta \ln \left[\frac{\sqrt{\left(x_{U}-x\right)^{2}+\left(y_{U}-y\right)^{2}+D_{1}^{2}}+D_{1}}{\sqrt{\left(x_{L}-x\right)^{2}+\left(y_{U}-y\right)^{2}+D_{1}^{2}}+D_{1}}\right]$

$+\cos I_{\cos } \cos \theta \sin \left[\frac{\sqrt{\left(x_{U}-x\right)^{2}+\left(y_{L}-y\right)^{2}+D_{1}^{2}}+D_{1}}{\sqrt{\left(x_{L}-x\right)^{2}+\left(y_{L}-y\right)^{2}+D_{1}^{2}}+D_{1}}\right]$ 
$-\cos I \cos I_{0} \cos \theta \tan ^{-1}\left[\frac{\left(x_{U}-x\right)\left(y_{U}^{-y}\right)}{\left(x_{U}-x\right)^{2}+D_{1} \sqrt{\left(x_{U}-x\right)^{2}+\left(y_{U}-y\right)^{2}+D_{1}^{2}}+D_{1}^{2}}\right]$

$+\cos I \cos I_{0} \cos \theta \tan ^{-1}\left[\frac{\left(x_{L}-x\right)\left(y_{U}-y\right)}{\left(x_{L}-x\right)^{2}+D_{1} \sqrt{\left(x_{L}-x\right)^{2}+\left(y_{U}-y\right)^{2}+D_{1}^{2}}+D_{1}^{2}}\right]$

$+\cos I \cos I_{0} \cos \theta \tan ^{-1}\left[\frac{\left(x_{U}-x\right)\left(y_{L}-y\right)}{\left(x_{U}-x\right)^{2}+D_{1} \sqrt{\left(x_{U}-x\right)^{2}+\left(y_{L}-y\right)^{2}+D_{1}^{2}}+D_{1}^{2}}\right]$

$-\cos I \cos I_{0} \cos \theta \tan ^{-1}\left[\frac{\left(x_{L}-x\right)\left(y_{L}-y\right)}{\left(x_{L}-x\right)^{2}+D_{1} \sqrt{\left(x_{L}-x\right)^{2}+\left(y_{L}-y\right)^{2}+D_{1}^{2}}+D_{1}^{2}}\right]$

$+\sin I \sin I_{0} \tan ^{-1}\left[\frac{\left(x_{U}-x\right)\left(y_{U}-y\right)}{D_{1} \sqrt{\left(x_{U}-x\right)^{2}+\left(y_{U}-y\right)^{2}+D_{1}^{2}}}\right]$

$-\sin I \sin I_{0} \tan ^{-1}\left[\frac{\left(x_{L}-x\right)\left(y_{U}-y\right)}{D_{1} \sqrt{\left(x_{L}-x\right)^{2}+\left(y_{U}-y\right)^{2}+D_{1}^{2}}}\right]$

$-\sin I \sin I_{0} \tan ^{-1}\left[\frac{\left(x_{U}-x\right)\left(y_{L}-y\right)}{D_{1} \sqrt{\left(x_{U}-x\right)^{2}+\left(y_{L}-y\right)^{2}+D_{1}^{2}}}\right]$ 
$\left.+\sin I \sin I_{0} \tan ^{-1}\left[\frac{\left(x_{L}-x\right)\left(y_{L}-y\right)}{D_{1} \sqrt{\left(x_{L}-x\right)^{2}+\left(y_{L}-y\right)^{2}+D_{1}^{2}}}\right]\right\}$

The inclination of the static inducing field, as measured from the surface, is represented by $I_{0}$, whereas the inclination and azimuth of the magnetization vector within the prismatic body are given by $I$ and $\theta$. For sensitivity analyses of a body of small thickness $T$, eq (8) may be used in a manner analogous to eq (7). In practice it is difficult to predict any component of permanent magnetization. However, approximate anomalous static field signatures can be predicted in this passive detection scheme for ranges of the susceptibility contrasts measured for most metallic mines and for various soil types. These anomalous signatures can be predicted for various horizontal offsets and heights of the sensor from the actual buried mine so as to indicate threshold sensitivities required in any actual field instrumentation.

\subsection{Sensitivity Analyses}

For most iron and steel objects a conservative range of the magnetic susceptibility $\mathrm{k}$ is between 1 and 10. Anderson [4] has reported background magnetic susceptibilities of various soil types, and the measured susceptibility of the U.S. Army BRDC magnetite-sand mine lane mixture is $350,000 \times 10^{-6}$. These values are summarized in Table 2. The contrasts in susceptibility between measured permeable soils and metallic mines or firing pins may be used in sensitivity analyses for either passive schemes (such as that described here) or active electromagnetic induction sensing. 
Table 2. Magnetic susceptibilities of various soil types (after Anderson [4]).

\begin{tabular}{|c|c|c|c|}
\hline LOCATION & SOIL TYPE & $\begin{array}{l}\text { PARENT ROCK } \\
\text { TYPE }\end{array}$ & $\frac{\mathrm{k}}{4 \pi}\left(\times 10^{6}\right)$ \\
\hline Fairfax Co., Va. & $\begin{array}{c}\text { Congarie silty } \\
\text { clay loam }\end{array}$ & Alluvial clay & 15 \\
\hline Fairfax Co., Va. & Susquehanna loam & Heavy coastal deposits & 75 \\
\hline Fairfax Co., Va. & Chester loam & $\begin{array}{l}\text { Granite, granite } \\
\text { gneiss and schist }\end{array}$ & 20 \\
\hline$\overline{\text { Panama Canal Zone }}$ & Coastal sands & $\begin{array}{l}\text { Basic ferromagnesian } \\
\text { igneous }\end{array}$ & $\begin{array}{r}50,000 \text { to } \\
150,000\end{array}$ \\
\hline$\overline{\mathrm{BRDC}}$ & $\begin{array}{c}\text { Magnetite-sand } \\
\text { mixture }\end{array}$ & & 27,800 \\
\hline
\end{tabular}

Table 2 shows that a minimal contrast in magnetic susceptibility between the highly magnetic Panama Canal Zone coastal sands and a steel clad metal mine is 10.68. For purposes of this sensitivity analysis the dimensions of the mine are taken to be $76 \mathrm{~mm} \times 76 \mathrm{~mm} \times 76 \mathrm{~mm}$, and the mine model is a solid with a minimal susceptibility contrast of 10.68 . The magnetization vector is also taken as vertical in an inducing field of $50000 \mathrm{nT}$.

\section{Relative anomalous magnetometric signatures for variable offsets and} heights of a total field head from the buried metallic mine are shown in figures 11, 12, and 13. Heights of the sensor head were taken as $0.3 \mathrm{~m}, 1.5 \mathrm{~m}$, and $3 \mathrm{~m}$, respectively. For a sensor head which is just $0.3 \mathrm{~m}$ above the burial depth of this modeled mine, the detection range is $2.4 \mathrm{~m}$. A threshold offset detection range for this mine size is given as a function of sensor height in figure 14. Clearly, a larger metallic mine would have a much greater detection range, while that of a permeable firing pin would be much less. 


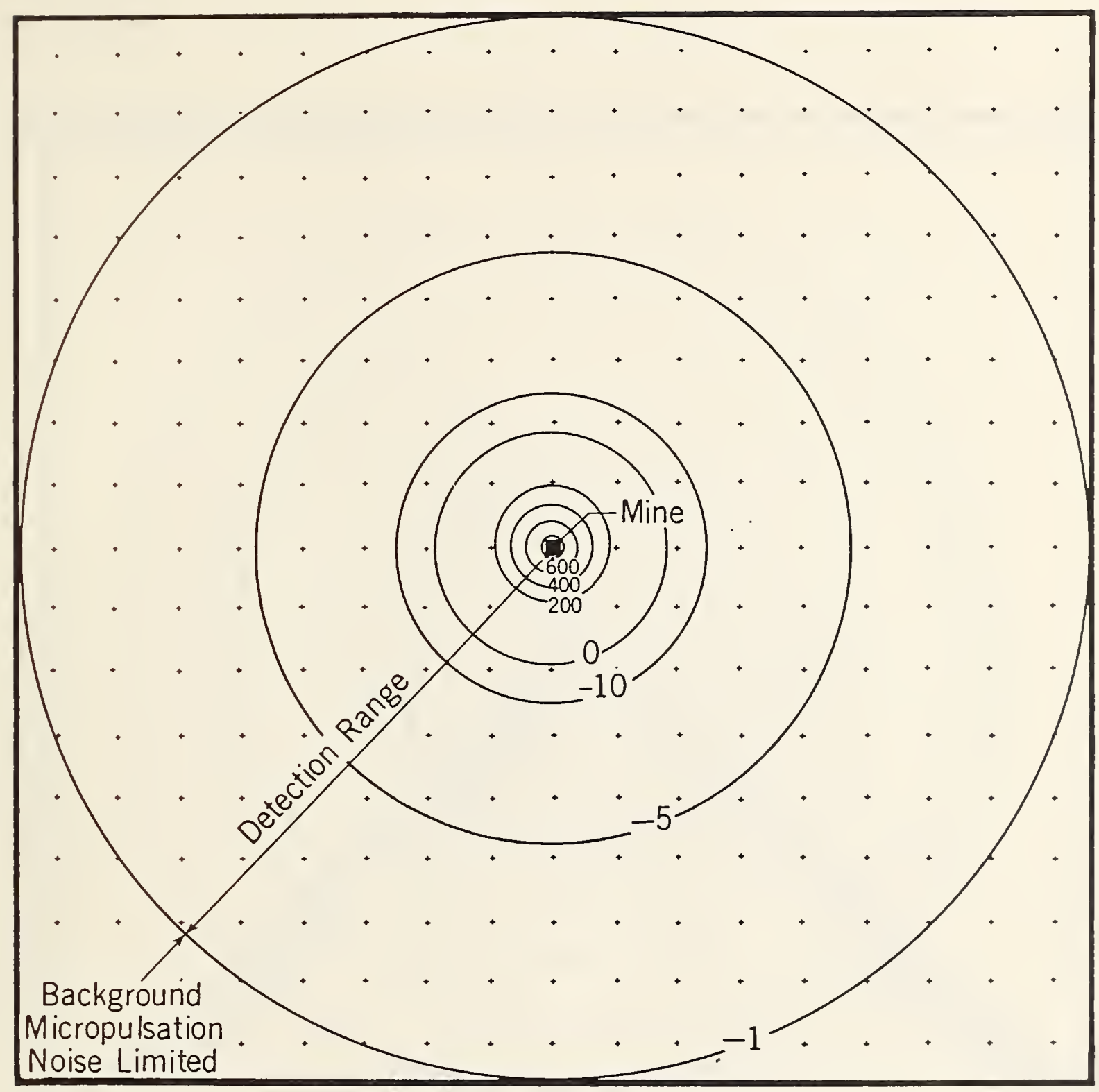

Applied Field: 50000 nT

Susceptibility Contrast: 10.68

Mine Dimensions: $76 \mathrm{~mm} \times 76 \mathrm{~mm} \times 76 \mathrm{~mm}$

Inclination of Polarization Vector: $90^{\circ}$

Sensor Height Above Mine: $0.3 \mathrm{~m}$

Contour Interval: Variable nT

Horizontal $=$ Vertical Scale

Figure 11. Anomalous total magnetic field intensity for passive magnetometric mine detection (magnetically permeable host medium). 


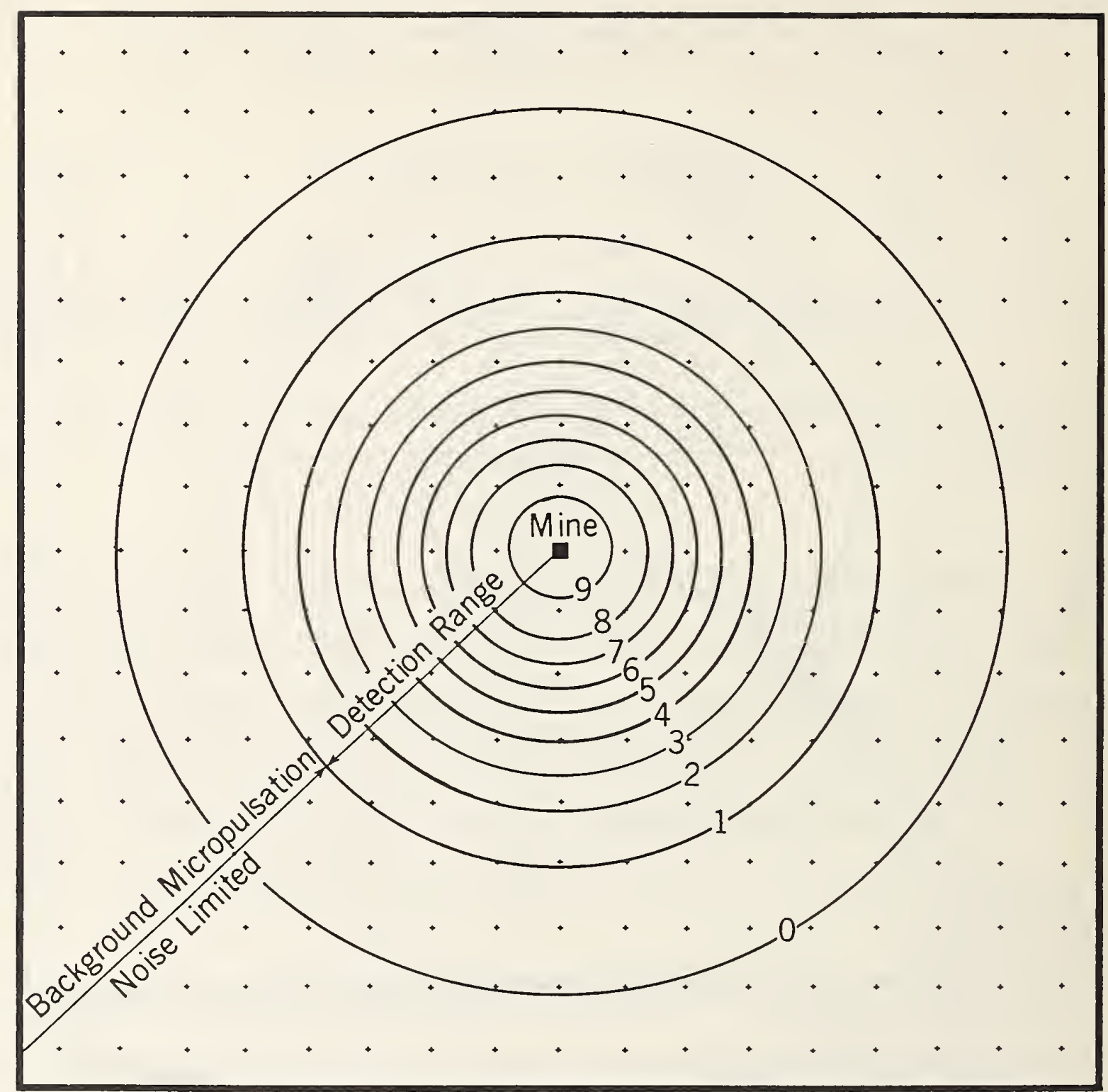

Applied Field: $50000 \mathrm{nT}$

Susceptibility Contrast: 10.68

Mine Dimensions: $76 \mathrm{~mm} \times 76 \mathrm{~mm} \times 76 \mathrm{~mm}$

Inclination of Polarization Vector: $90^{\circ}$

Sensor Height Above Mine: $1.5 \mathrm{~m}$

Contour Interval: $1.0 \mathrm{nT}$

Horizontal $=$ Vertical Scale

Figure 12. Anomalous total magnetic field intensity for passive magnetometric mine detection (magnetically permeable host medium). 


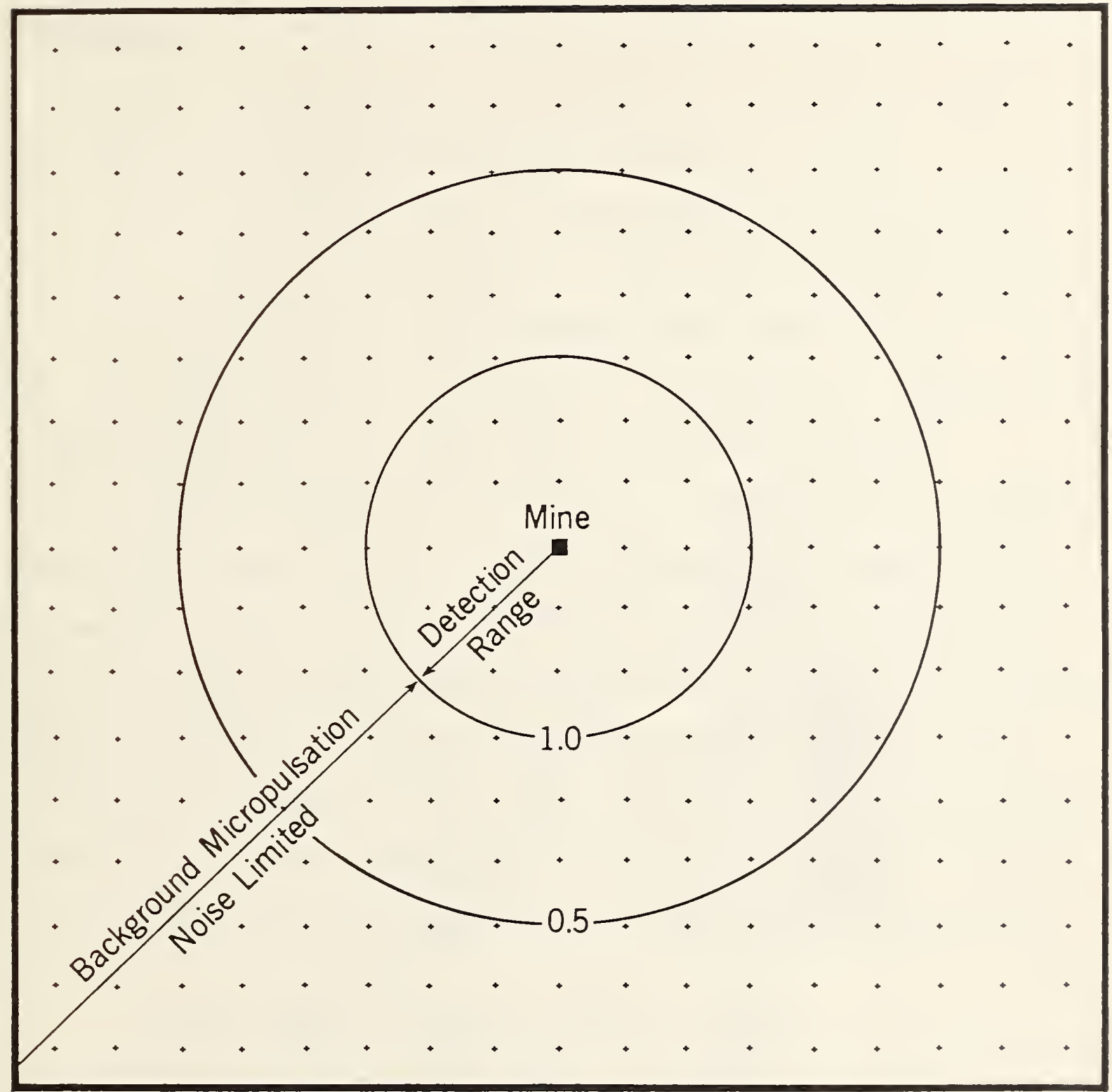

Applied Field: 50000 nT

Susceptibility Contrast: 10.68

Mine Dimensions: $76 \mathrm{~mm} \times 76 \mathrm{~mm} \times 76 \mathrm{~mm}$

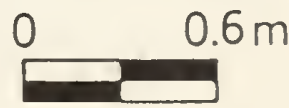

Inclination of Polarization Vector: $90^{\circ}$

Sensor Height Above Mine: $3 \mathrm{~m}$

Contour Interval: $0.5 \mathrm{nT}$

Horizontal $=$ Vertical Scale

Figure 13. Anomalous total magnetic field intensity for passive magnetometric mine detection (magnetically permeable host medium). 


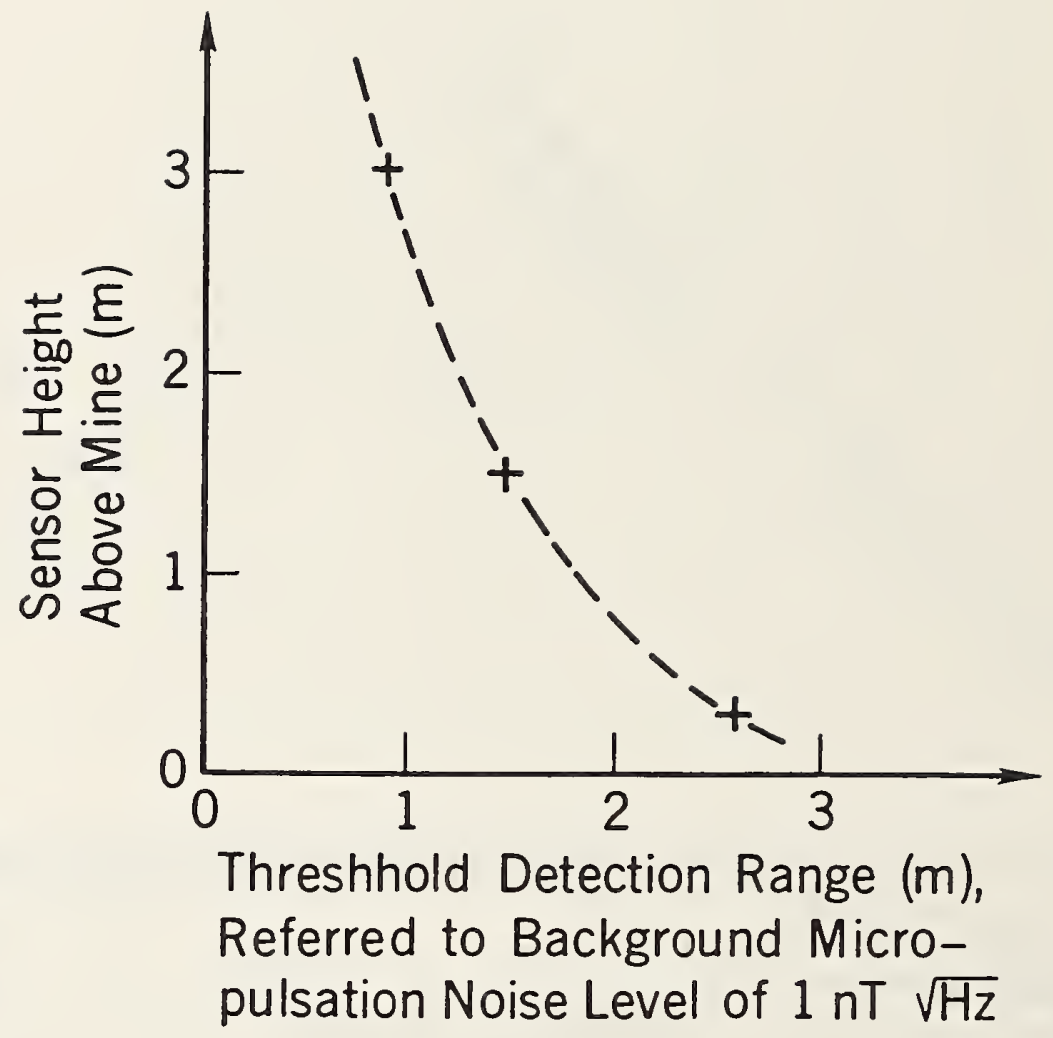

Figure 14. Threshold offset detection range versus sensor height for $7.6 \mathrm{~cm} \times$ $7.6 \mathrm{~cm} \times 7.6 \mathrm{~cm}$ buried magnetic mine. 


\section{CONCLUSIONS}

The magnetic susceptibility of soils depends on the component magnetic minerals derived from chemical and mechanical breakdown of bedrock. Magnetic minerals of importance are few, and those most commonly encountered are the iron and titanium oxides. One measurement technique for determining material magnetic susceptibility is to observe the inductance change required to balance a bridge circuit when the sample under test is inserted into a solenoidal test coil composing one arm of the circuit. The inductance adjustment is proportional to the susceptibility of the sample, and calibration is achieved by balancing the bridge with known weights of a paramagnetic compound such as ferrous ammonium sulfate. In order to avoid a conductivity response of the sample in the weak time-varying source field of the bridge test coil, a maximum allowable test frequency is chosen so that the induction number within the sample under test is much less than 1. This criterion can be deduced from an examination of the in-phase and quadrature components of the induced dipole moment of a conductive permeable sphere in a uniform alternating magnetic field.

Magnetic susceptibility and conductivity measurements provide practical property range limits from which sensitivity analyses for active or passive metal detection systems may be performed. Examination of the induced dipole moment of a conductive, permeable sphere, placed in a permeable medium and excited by a uniform alternating magnetic field, also reveals that the operating frequency of an active detection system can be chosen so that the quadrature response is maximized for a given diameter of the buried sphere. This size discrimination capability is possible when the relative magnetic 
permeability contrast between the target sphere and enclosing soil medium is known, as well as the conductivity of the sphere.

An example of the use of magnetostatic measurements for passive detection of buried metallic mines has been considered which allows an arbitrary geometry of the mine in an arbitrary inducing field and in a magnetically permeable background medium. Typical commercial proton precession magnetometers have tuning sensitivities permitting total magnetic field intensity measurements accurate to $\pm 0.5 \mathrm{nT}$. In a field of $50000 \mathrm{nT}$ and time-varying ambient noise levels (over the tuning period) of $\pm 0.5 \mathrm{nT}$, a metallic mine $7.6 \mathrm{~cm}$ on a side would have an anomalous signature of $1000 \mathrm{nT}$ at zero offset and would still be detectable (over background noise levels) at offset distances of about $2 \mathrm{~m}$ for a sensor height of only $0.3 \mathrm{~m}$. However, at sensor heights of $3 \mathrm{~m}$ the presence of a mine $7.6 \mathrm{~cm}$ on a side would be barely detectable, giving a maximum anomalous total magnetic field strength at zero offset of only $1.3 \mathrm{nT}$.

Although not performed here, the same type of sensitivity analyses for electromagnetic induction detection schemes can be made on the basis of magnetic permeability and electrical conductivity measurements. The total magnetic field signatures due to metallic firing pins would be lost in ambient noise levels. However, the very low inherent noise levels of superconducting quantum interference device (SQUID) sensors suggest that SQUID magnetic gradiometers might be a useful detection tool for such a case. The quantity measured by such a system will be the gradient tensor, the spatial rate of change of the vector components of the magnetic field. The resulting tensor components could then be downward continued to accentuate anomalous 
signatures, and it may be possible to invoke depth estimation procedures to filter out magnetic surface clutter. These problems are worthy of further theoretical and experimental study. 
8. REFERENCES

[1] Nagata, T. Rock magnetism. Tokyo: Maruzen Press; 1961. 350 p.

[2] Akimoto, S. Magnetic properties of ferromagnetic oxide minerals as a basis of rock magnetism. Adv. Phys. 6(288); 1957.

[3] Heiland, C.A. Geophysical exploration. Englewood Cliffs, New Jersey: Prentice Hall; 1940. p. 310-314.

[4] Anderson, L. Private communication. U.S. Army Belvoir RD \& E Center, Countermine Systems Directorate, Fort Belvoir, VA 22060-5606; 1987.

[5] Ward, S.H. Electromagnetic theory for geophysical applications. Mining Geophys. II: 78; 1967.

[6] Wait, J.R. A conducting sphere in a time varying field. Geophys. $16: 666 ; 1951$.

[7] Geoinstruments, model JH-8*.

*Certain commercial equipment, instruments, or materials are identified in this in order to specify the experimental procedure. Such identification does not imply recommendation or endorsement by the National Institute of Standards and Technology nor does it imply that the materials or equipment identified are necessarily the best available for the purpose. 
NBS-114A (AEV. 2-8C)

U.S. DEPT. OF COMM.

BIBLIOGRAPHIC DATA

SHEET (See inseructions)

4. TITLE AND SUBTITLE

1. PUBLICATION OR REPORT NO.

NISTIR $88-3098$
2. Performing Organ. Report No. 3. Publication Date

October 1988

Magnetostatic MEASUREMENTS FOR MINE DETECTION

5. $A \cup T H O R(S)$

Richard G. Geyer

6. PERFORMING ORGANIZATION (If joint or other than NBS, see instructions) National Institute of Standards and Technology NAFHONAL-BUREAU-OFSFANBAROS DEPARTMENT OF COMMERCE WASHINGTON, D.C. 20234

7. Contract/Grant No.

8. Type of Report \& Period Covered

9. SPONSORING ORGANIZATION NAME AND COMPLETE ADDRESS (Street, City, State, ZIP)

U.S. Army Belvoir Research and Development Center

Fort Belvoir, Virginia 22060-5606

10. SUPPLEMENTARY NOTES

[] Document describes a computer program; SF-185, FIPS Software Summary, is attached.

11. ABSTRACT (A 200-word or less factual summary of most significant information. If document includes a significant bibliography or literature survey. mention it here)

The use of a Maxwell inductance bridge and calibration procedure for measuring the magnetic susceptibility of magnetically linear, homogeneous, and isotropic materials are reviewed. A complication in this measurement exists since electromagnetic induction sensors respond to the product of the magnetic permeability and electrical conductivity. For this reason, frequency limitations resulting from sample size and conductivity must be considered. Such limitations can be specified by examining the in-phase and quadrature components of the induced dipole moment of a conductive, permeable sphere of diameter equivalent to that of the bridge test coil in a uniform alternating magnetic field and by choosing a maximum allowable test frequency that gives an induction number much less than 1 within the sphere.

Magnetic susceptibility measurements are applied to the passive magnetometric detection problem of an arbitrarily shaped susceptible (metallic) mine buried in a magnetically permeable earth. For analysis purposes a conservative susceptibility contrast between a typical metallic mine and host soil having the same measured magnetic characteristics as the U.S. Army Belvoir Research and Development Center (BRDC) magnetite-sand mine lane mixture was assumed. Anomalous detection limits were then calculated for various total field intensity (Proton precession) sensor head heights and offset distances, given mine dimensions as small as $7.6 \mathrm{~cm}$ on a side.

12. KEY WORDS (Six to twelve entries; alphabetical order: capitalize only proper names; and separate key words by semicolons) inductance bridge; magnetic permeability; magnetic susceptibility; magnetometric detection; magnetostatic

\section{AVAILABILITY}

[x] Unlimited

[ For Official Distribution. Do Not Release to NTIS $\square$ Order From Superintendent of Documents, U.S. Government Printing Office, Washington, D.C.
20402 .

14. NO. OF

PRINTED PAGES

36

15. Price

X Order From National Technical Information Service (NTIS), Springfield, VA. 2216I 


